\title{
Statistics of Random Surfaces ${ }^{1}$
}

\author{
I. Kay and P. Swerling \\ Contribution From Conductron Corporation, Ann Arbor, Mich., Inglewood, Calif.
}

(Received December 6, 1963; revised March 20, 1964)

This paper is presented in two distinct parts.

Part 1 begins by giving a number of examples of random surfaces and summarizing the various applications of random surface theory; including applications to

(a) electromagnetic scattering from such surfaces,

(b) the information content of photographs, maps, etc. interpreted as random surfaces, and implications for bandwidth compression.

This part of the paper will also summarize the major results which have previously been obtained, and will outline the approaches used to obtain these results. Finally, some interesting additional problems in this field are stated.

Part 2 of the paper is devoted to new results on the properties of random spherical surfaces, and the application to electromagnetic scattering from such surfaces. These results primarily concern the statistics of the number of specular points per unit surface area.

The detailed elaboration of the mathematical approaches to obtaining the major results of random surface theory, and even the exhibiting of the major formulas, would be very complex and space consuming. Therefore, insofar as previously obtained results are concerned, we will merely give outlines and examples, with references to the appropriate literature for the details. Such detailed mathematics as appears in this paper is reserved for the new results presented in part 2.

For convenience, the two parts have separate numbering for equations and references.

\section{Part 1-Outline of Random Surface Theory and Applications}

\section{Introduction}

\subsection{Definition and Fxamples}

A random surface is a random process $\{f(x, y)\}$ depending (usually) on two real parameters $x$ and $y$. Although we have used the notation $(x, y)$, these do not necessarily represent rectilinear Cartesian coordinates.

For example, one of the parameters may represent time: $y=t$. In this case, we may be dealing with a random function of one spatial parameter which is also a random function of time. However, the mathematical techniques are those applicable to random surfaces.

Also, the parameters, even if they represent spatial coordinates, may represent coordinates other than Cartesian. For example, we may have $x=\theta, y=\phi$, where $\theta, \phi$ are spherical angular coordinates.

Occasionally, we may be dealing with a random function of two spatial coordinates which is also a random function of time, in which case the random surface in question must be represented as a stochastic process $\{f(x, y, t)\}$ depending on three real parameters.

Examples of random surfaces are:

(a) The surface of the ocean or other body of water (in which $f$ represents level with respect to a mean level),

(b) a topographic map ( $f$ represents altitude),

(c) an atmospheric pressure chart ( $f$ represents atmospberic pressure),

(d) a photograph or television picture ( $f$ represents brightness),

1 A portion of the work reported on in this paper was supported by the National Aeronautics and Space Administration under Contract NASA 490 . 
(e) an antenna pattern in two spherical angular coordinates, and

(f) a rough spherical surface, such as that of the moon or other planet considered as a whole.

All of the above, but in particular, (a), (c), and (d), may depend not only on two spatial coordinates but on time as well.

As previously stated, random functions of two parameters, one of which represents a spatial coordinate and the other time (as in a moving waveform) can also be considered to be random surfaces from the point of view of statistical treatment.

\subsection{Applications}

The most extensive treatment of random surfaces remains that of the series of papers of Longuet-Higgins $[1952 ; 1956 ; 1957 \mathrm{a}, \mathrm{b}, \mathrm{c}]$. Much of this was motivated by applications to the surface of the sea.

In particular, many of the properties studied are of interest for studying the electromagnetic (radar or optical) scattering properties of the sea. For example, the statistics of specular points and their motion are properties affecting the intensity and spectrum of scattered energy in a given direction.

Clearly, this application to electromagnetic scattering properties is not restricted to the surface of the sea, but may be relevant to scattering from any object which can reasonably be represented as a random surface. Part 2 of this paper deals with the statistics of specular points on a random spherical surface, and was motivated by problems of reflection of radar waves from the moon.

Another major application of random surface theory is to the information content of various examples of random surfaces (in particular, photographs and television pictures but also including, possibly, topographic maps, pressure charts, antenna patterns, etc.).

By information content is meant how many bits of information are required to describe such a surface to some given degree of accuracy. This affects the storage requirement for storing large amounts of data of this type, as well as the bandwidth requirements for transmitting such data through a communication channel. The work of Swerling [1962] on contours of random surfaces was motivated by these considerations.

If the statistics of the random surface are completely specified, the information content can be determined according to the standard formulas of information theory [Swerling, 1962]. However, bounds on the information content may be more easily obtainable by studying certain statistical properties of contours, such as expected length per unit area, expected number of separate closed contour pieces, and expected "roughness" of contours.

The above discussion is by no means exhaustive and is in fact only a sketchy outline of the possible applications of random surface theory and of the particular properties of random surfaces which are of interest in these applications. For example, extensive study has been made of the statistics of the number and velocity of maxima and minima of moving waveforms, the velocity of the crossings of any given level, the annihilation and creation of specular points, etc. [Longuet-Higgins, 1952; 1956; 1957 a, b, c].

\section{Analytical Techniques for Random Surfaces}

The statistical properties of random surfaces which have been most extensively studied involve surface properties which can be described in terms of the differential geometry of the surface - that is, in terms of the values taken on by the function $f$ and its partial derivatives of various orders.

These properties involve the probability that the function $f$ and its partial derivatives take on certain values or that the line integrals, along contours, take on certain functions of the partial derivatives.

For example, an extreme point of a random surface is characterized by $f_{x}=0, f_{y}=0 . \quad A$ contour is characterized by $f=z_{0}$. In a moving waveform $f(x, t)$, the velocity of a point at a 
constant level $f=z_{1}$ is the value of $f_{t} / f_{x}$ subject to $f=z_{1}$. Specular points can be defined as points at which the gradient has a definite value. Such examples can be multiplied indefinitely.

Thus, the approach to deriving the desired statistics of these surface properties consists first in describing the phenomenon in question in terms of the values of $f$ and its partial derivatives, and then taking the appropriate statistical average. This will be illustrated by several specific examples below.

The results depend on the joint probability density function ( $p d f$ ) of $f$ and its partial derivatives. It is worth noting that most of the results which have been obtained involve the joint $p d f$ of the function and its partials evaluated at the same point. However, as will be pointed out, many interesting statistical properties involve the joint $p d f$ of the function and its partials evaluated at two or more different points; these properties have not been extensively treated.

As a first example, consider the statistical properties of the length of contours in a planar surface. Following Swerling [1962], let the intersection of the surface $z=f(x, y)$ with a plane $z=z_{0}$ be called a $z_{0}$-contour. Let $\nabla f$ denote the gradient, that is the vector having components $f_{x}, f_{y}$.

Define

$$
\begin{aligned}
F_{d}(u, v) & =\frac{1}{d} \text { if }|u-v| \leq \frac{d}{2} \\
& =0 \text { if }|u-v|>\frac{d}{2}
\end{aligned}
$$

Then, the total length $L_{R}\left(z_{0}\right)$ of the $z_{0}$-contour in a region $R$ of the $(x, y)$-plane is

$$
L_{R}\left(z_{0}\right)=\lim _{d \rightarrow 0} \int_{R}|\nabla f| F_{d}\left(f, z_{0}\right) d x d y .
$$

Equation (2) gives an expression for $L_{R}\left(z_{0}\right)$ apart from any statistical considerations. It is worth noting that this expression depends on the fact that we are dealing with planar surfaces rather than, say, spherical surfaces in this example. From this expression one can derive the desired statistical properties of $L_{R}\left(z_{0}\right)$ in terms of those of $f$.

For example, denoting expected values by $E(\quad)$, we have

$$
E\left[L_{R}\left(z_{0}\right)\right]=\int_{R} \int \sqrt{u_{1}^{2}+u_{2}^{2}} W\left(z_{0}, u_{1}, u_{2} \mid x, y\right) d u_{1} d u_{2} d x d y
$$

where $W\left(u_{0}, u_{1}, u_{2} \mid x, y\right)$ is the joint $p d f$ of $f, f_{x}$, and $f_{y}$, all evaluated at $(x, y)$. In (3), the inner integral is extended over $-\infty<u_{1}, u_{2}<\infty$. If $\{f(x, y)\}$ is stationary, $W$ is independent of $x, y$; the expected length per unit area is obtained by dropping the integral over $R$.

A second example involves events taking place at isolated points rather than along a whole contour, namely, the expected number of horizontal tangents to a $z_{0}$-contour. Such a tangent occurs when $f=z_{0}, f_{x}=0$.

The number of points within $R$ where $f=z_{0}, f_{x}=0$ is

$$
T_{R}\left(z_{0}\right)=\lim _{d \rightarrow 0} \int_{R}\left|f_{x} f_{x y}-f_{y} f_{x x}\right| f_{d}\left(f_{x}, 0\right) F_{d}\left(f, z_{0}\right) d x d y,
$$

and the expected number in $R$ is

$$
E\left[T_{R}\left(z_{0}\right)\right]=\int_{R} \int\left|u_{2} u_{3}\right| W\left(z_{0}, 0, u_{2}, u_{3} \mid x, y\right) d u_{2} d u_{3} d x d y,
$$

where $W\left(u_{0}, u_{1}, u_{2}, u_{3} \mid x, y\right)$ is the joint $p d f$ of $f, f_{x}, f_{y}$, and $f_{x x}$ at the point $x, y$. 
A third example involves line integrals of the curvature of a contour. Let a $z_{0}$-contour be expressed in parametric form $x(s), y(s)$. The line integral along the contour of $\left(\theta^{\prime}(s)\right)^{2}$ is a measure of the roughness of the contour, where $\theta(s)$ is the angle of a tangent to a contour.

Then [Swerling, 1962],

$E\left[\int\left[\theta^{\prime}(s)\right]^{2} d s\right]=\int_{R} \int\left(u_{1}^{2}+u_{2}^{2}\right)^{-5 / 2}\left(u_{1}^{2} u_{5}-2 u_{1} u_{2} u_{4}+u_{2}^{2} u_{3}\right)^{2}$

where

$$
\times W\left(z_{0}, u_{1}, u_{2}, u_{3}, u_{4}, u_{5} \mid x, y\right) d u_{1} \ldots d u_{5} d x d y
$$

$$
W\left(u_{0}, u_{1}, u_{2}, u_{,_{3}} u_{4}, u_{5} \mid x, y\right) \text { is the joint } p d f \text { of } f, f_{x}, f_{y}, f_{x x}, f_{x y} \text {, and } f_{y y} \text { at } x, y \text {. }
$$

The integral on the left side of (6) is the line integral along the $z_{0}$-contour within $R$.

Expression (6) arises from the fact that $\theta^{\prime}(s)$ can be expressed in terms of the partials of $f$ as follows:

$$
\theta^{\prime}(s)=-\left\{\frac{f_{x}^{2} f_{y y}-2 f_{x} f_{y} f_{x y}+f_{y}^{2} f_{x x}}{|\nabla f|^{3}}\right\} .
$$

These examples are sufficient to illustrate the procedure: first express the surface property in terms of the appropriate conditions on the function $f$ and its partials; then, find a nonstatistical expression for the number of points at which the property holds, the length of the contour, or whatever may be the precise quantity for which one wishes to derive statistics; this expression can then be used to derive the statistical properties of this quantity, such as its expected value.

For gaussian random surfaces, the expressions in terms of the joint $p d f$ 's, $W$ can often be explicitly evaluated. The probability distributions for gaussian surfaces can be specified by specifying the function

$$
\varphi(\xi, \eta \mid x, y)=E[f(x+\xi, y+\eta), f(x, y)] .
$$

(If the process is stationary, the dependence on $x, y$ disappears.)

Further, all of the joint $p d f$ 's, $W$ can be explicitly written down in terms of the function $\varphi$ and its partial derivations with respect to $\xi$ and $\eta$, evaluated at $\xi=0, \eta=0$.

The reader is referred to the literature (references, Part 1) for the resulting expressions.

\section{Additional Problems}

A number of additional problems of interest remain to be treated in the field of random surfaces.

One such problem is that of extending the results to nonplanar random surfaces, such as spherical surfaces. Part 2 of this paper gives new results on this precise problem.

As previously noted, the specific form taken by the expressions depends on the differential geometry of the surface in question.

A second problem involves deriving the higher order moments rather than just expected values. This problem can easily be attacked in principle by utilizing the exact (nonstatistical) expressions for the quantities in question e-g., (2) and (4).

As an illustration, suppose it is desired to derive the second moment of $L_{R}\left(z_{0}\right)$, the length of a $z_{0}$-contour in $R$. From (2),

$$
\left[L_{R}\left(z_{0}\right)\right]^{2}=\lim _{d, d^{\prime} \rightarrow 0} \int_{R} \int_{R}|\nabla f(x, y)|\left|\nabla f\left(x^{\prime}, y^{\prime}\right)\right| F_{d}\left[f(x, y), z_{0}\right] F_{d^{\prime}}\left[f\left(x^{\prime}, y^{\prime}\right), z_{0}\right] d x d y d x^{\prime} d y^{\prime}
$$

Then, $E\left[L_{R}\left(z_{0}\right)\right]^{2}$ can be obtained directly from (9). The resulting expression involves the joint probability function of $f(x, y), f_{x}(x, y), f_{y}(x, y), f\left(x^{\prime}, y^{\prime}\right), f_{x}\left(x^{\prime}, y^{\prime}\right), f_{y}\left(x^{\prime}, y^{\prime}\right)$, for all pairs of points $(x, y)$ and $\left(x^{\prime}, y^{\prime}\right)$. It is very difficult to evaluate explicitly even for stationary gaussian surfaces. 
Similarly, higher moments may be derived by expressing the powers of $L_{R}\left(z_{0}\right)$ as multiple integrals and taking expected values.

The same remarks apply to other quantities for which the higher moments may be desired.

A third category of problems of significance is the further study of the contours regarded as random processes. A connected piece of a contour can be expressed in the parametric form $x(s), y(s)$ where $s=$ arc length. The derivatives $x^{\prime}(s), y^{\prime}(s)$ are related; in fact, $y^{\prime}(s)$ is determined up to sign by $x^{\prime}(s)$. The statistical properties of $x^{\prime}(s)$ or $y^{\prime}(s)$ regarded as random processes are of interest. Alternatively, one could study $\theta(s), \theta=$ angle of tangent to a contour, regarded as a random process; $\theta(s)$ is a function of $x^{\prime}(s)$ and $y^{\prime}(s)$.

Swerling [1962] derives an expression for the expected value of the line integral along a contour of $\left[\theta^{\prime}(s)\right]^{2}$. Similarly, expressions can be derived for the expected value of the line integral of $\left[x^{\prime \prime}(s)\right]^{2}$ or $\left[y^{\prime \prime}(s)\right]^{2}$. It is pointed out by Swerling [1962] that these expected values can be used to define quantities analogous to the second moment of the spectral density function of $\theta(s)$ or $x^{\prime}(s)$, assuming that such spectral density functions could be defined rigorously.

In exactly the same way, expected values of line integrals of the squares of higher derivatives of $\theta(s)$ or $x^{\prime}(s)$ can be obtained, since these higher derivatives can be expressed as functions of the partial derivatives of $f$. These would be analogous to higher moments of the spectral density function. However, they would be very difficult to evaluate explicitly.

Apart from explicit evaluations of the expected values of line integrals of this type, the rigorous definition of concepts corresponding to spectral density function for the contours, regarded as random processes, is a problem of interest.

\section{References (Part 1)}

Longuet-Higgins, M. S. (1952), On the statistical distribution of the heights of sea-waves, J. Marine Res. 9, $245-266$.

Longuet-Higgins, M. S. (1956), Statistical properties of a moving wave-form, Proc. Cambridge Phil. Soc. 52, $234-245$.

Longuet-Higgins, M. S. (1957a), Statistical properties of an isotropic random surface, Phil. Trans. Roy. Soc. London A250, 157-174.

Longuet-Higgins, M. S. (1957b), The statistical analysis of a random moving surface, Phil. Trans. Roy. Soc. London A249, 321-387.

Longuet-Higgins, M. S. (1957c), On the velocities of the maxima in a moving wave-form, Proc. Cambridge Phil. Soc. 53, 230-233.

Swerling, P. (1962), Statistical properties of the contours of random surfaces, IRE Trans. Inform. Theory IT-8, 315-321.

\section{Part 2-Distribution of Specular Points on a Rough Spherical Surface}

\section{Introduction}

The problem of describing the scattering of high frequency electromagnetic or acoustic waves by a statistically rough spherical surface is the primary physical motivation for the analysis presented in this article. The theory of scattering from rough surfaces can be traced back to the work of Rayleigh [1929], who was interested in the reflection of sound from statistically rough planes. In comparatively recent times a great deal of attention has been paid to the theoretical treatment of similar phenomena involving electromagnetic waves as well as acoustical waves, and the subject has been developed to the point where it is now regarded as one of the more important aspects of applied scattering theory.

While many investigators have contributed directly to this subject, both theoretically and experimentally, the present article represents an attempt to follow a line of research begun by M. S. Longuet-Higgins $[1952 ; 1956 ; 1957$ a, b, c] on a more general class of problems. 
That work, which has been extended further by P. Swerling [1962], ${ }^{2}$ involves the statistical description of random surfaces and the resulting geometrical effects.

Among the questions considered by Longuet-Higgins is the description of those surface properties which affect the reflection of coherent unpolarized light according to the theory of geometrical optics. He has discussed, for example, the distribution of specular points and twinkling effects of a moving sea in relation to certain moments of the surface power spectral density [Longuet-Higgins, 1957a]. Although both stationary and moving surfaces were considered, the investigations were confined to those cases in which the mean surface is a plane.

The present article will be concerned with the distribution of specular points for geometrical optics scattering by a statistically perturbed sphere. The problem will be restricted to the case of a source located at a distance which is large compared to the radius of the sphere. The procedure followed in the analysis is essentially that of Longuet-Higgins and Swerling.

An important example of the possible physical significance of the problem treated here is in the interpretation of radar scattering data resulting from earth based radar soundings of the moon. A complete statistical description of the specular return would also require a consideration of the distribution of Gaussian curvature over the lunar surface and, because of the coherent nature of the signals used, an analysis of the distribution of the path differences associated with the various specular point contributions. However, even the present investigation, restricted as it is to the relation between the fundamental properties of the perturbed spherical surface geometry and the distribution of specular points alone, should provide a certain amount of insight into the nature of the scattering and help to clarify some unresolved questions which have been raised concerning the lunar scattering datainterpretation. In particular, the theory developed here may help eventually to supply the relevant connection between the probable lunar surface geometry and the theory of Senior and Siegel [1960], in which it has been estimated that between 25 and 30 specular points exist near the moon's leading edge.

\section{Surface Angular Correlation Function}

Since the mean surface of the scattering object is a sphere it will be convenient to use standard spherical coordinates $r, \theta, \phi$ to describe the detailed surface geometry. Thus, if $\mathbf{k}$ is a fixed unit vector in the direction of the polar angle $\theta=0$, the angle $\phi$ will be measured from a fixed radius in the plane through the origin of the coordinate system and perpendicular to $\mathbf{k}$. The angle $\theta$ is formed by a radius vector $\mathbf{r}$ and a radial line in the direction of $\mathbf{k}$.

We shall assume that the given closed scattering surface is a random sample from a population of surfaces for which the mean surface is a fixed sphere of radius $\rho$, having its center at the origin of the coordinate system.

The population of surfaces can be represented by a stochastic variable of the form

$$
r(\theta, \varphi)=h(\theta, \varphi)+\rho,
$$

where $h(\theta, \varphi)$ is a random quantity having the mean value zero.

It will be convenient for obvious geometrical reasons to regard $h(\theta, \varphi)$ as represented by an expansion in spherical harmonics

$$
h(\theta, \varphi)=\sum_{n=0}^{\infty} A_{n} P_{n}(\cos \theta)+\sum_{n=1}^{\infty} \sum_{m=1}^{n}\left(A_{m n} \sin m \varphi+B_{m n} \cos m \varphi\right) P_{n}^{m}(\cos \theta) .
$$

It will be assumed that the coefficients $A_{m n}$ and $B_{m n}$ are independent random variables having zero means:

$$
E\left(A_{m n}\right)=E\left(B_{m n}\right)=0 \text { for all } m, n
$$




$$
\begin{gathered}
E\left(A_{m n} A_{m^{\prime} n^{\prime}}\right)=E\left(B_{m n} B_{m^{\prime} n^{\prime}}\right)=0 \text { for all } m, n, m^{\prime}, n^{\prime} \text { except } m=m^{\prime} \text { and } n=n^{\prime} ; \\
E\left(A_{m n} B_{m^{\prime} n^{\prime}}\right)=0 \text { for all } m, n, m^{\prime}, n^{\prime} ;
\end{gathered}
$$

in the above relations $A_{0 n}$ and $B_{0 n}$ are to be identified with $A_{n}$ and $B_{n}$. These assumptions are analogous to those which have been made in connection with the planar rough surface scattering problem (cf. [Rayleigh, 1929; Longuet-Higgins, 1952, 1956, 1957a; Swerling, 1962; Rice, 1951]), wherein a similar formalism based on a Fourier series representation of the perturbations is ordinarily used. In the present case the polar singularity of spherical coordinate representations dictates the use of the more appropriate spherical harmonics.

We can define the correlation function relative to $h(\theta, \varphi)$ for every pair of surface points, determined by the angular coordinates $\theta, \varphi$, and $\theta^{\prime}, \varphi^{\prime}$, as

$$
\begin{aligned}
E\left(h(\theta, \varphi) h\left(\theta^{\prime}, \varphi^{\prime}\right)\right)=\sum_{n=0}^{\infty} E\left(A_{n}^{2}\right) P_{n}(\cos \theta) P_{n}\left(\cos \theta^{\prime}\right) & +\sum_{n=1}^{\infty} \sum_{m=1}^{n}\left(E\left(A_{m n}^{2}\right) \sin m \varphi \sin m \varphi^{\prime}\right. \\
& \left.+E\left(B_{m n}^{2}\right) \cos m \varphi \cos m \varphi^{\prime}\right) P_{n}^{m}(\cos \theta) P_{n}^{m}\left(\cos \theta^{\prime}\right) .
\end{aligned}
$$

With one additional assumption it will follow that the distribution of $h(\theta, \varphi)$ is isotropic; that is, the correlation function $E\left(h(\theta, \varphi) h\left(\theta^{\prime}, \varphi^{\prime}\right)\right)$ is a function only of the angle $\gamma$ between the points $(\theta, \varphi)$ and $\left(\theta^{\prime}, \varphi^{\prime}\right)$. The angle $\gamma$ satisfies the relation

$$
\cos \gamma=\cos \theta \cos \theta^{\prime}+\sin \theta \sin \theta^{\prime} \cos \left(\varphi-\varphi^{\prime}\right) .
$$

Because of the uniqueness of the spherical harmonic expansion, the required additional assumption is

$$
E\left(A_{m n}^{2}\right)=E\left(B_{m n}^{2}\right)=2 \frac{(n-m) !}{(n+m) !} W_{n}
$$

the $W_{n}$ defined by (5) are coefficients in the expansion

$$
E\left(h(\theta, \varphi) h\left(\theta^{\prime}, \phi^{\prime}\right)\right)=\sum_{n=0}^{\infty} W_{n} P_{n}(\cos \gamma)=K(\cos \gamma)=K(\mu), \quad(\mu=\cos \gamma) .
$$

The fact that the relations (5) must hold when the distribution of $h(\theta, \varphi)$ is isotropic follows from an application of the addition theorem for spherical harmonics to (6) and a comparison of the coefficients with those of like terms in (3). ${ }^{3}$

The coefficients $W_{n}$ may be referred to collectively as the spherical spectral power density by analogy with the planar rough surface problem. In particular we have from (6) and the definition of $\gamma$ an analogous relation for the mean square surface devistion, which we shall refer to as the zeroth moment $M_{0}$ :

$$
E\left(h^{2}(\theta, \varphi)\right)=\sum_{n=0}^{\infty} W_{n}=M_{0}
$$

\section{Distribution of Specular Points}

As usual we shall assume that $h(\theta, \varphi)$ is a gaussian distributed random variable. The probability distribution of $h(\theta, \varphi)$ is then determined completely by the quantity $M_{0}$ defined in (7). Moreover, the joint probability distributions of $h(\theta, \varphi)$ and its derivatives with respect to $\theta$ and $\varphi$ of various orders are determined in terms of moments of the power spectral density function $K(\mu)$ defined by (6) (cf. [Swerling, 1962]). Probability distributions for geometrical properties of the surface can thus be calculated by the methods described in Swerling's paper. ${ }^{4}$

${ }^{3}$ The authors are grateful to the referee for pointing out that (6) also implics our other assumptions concerning the correlations of the $A_{m n}$ and $B_{m n}$.

${ }_{4}$ These methods are outlined in part I of this paper. 
The particular example of the procedure to be considered here is the derivation of the distribution of specular points for the scattering of waves by the surface when the source is located at a large distance compared to the radius $\rho$ of the mean sphere. In this connection it will also be assumed that $\rho$ is large compared to the wavelength, so that the dominant scattering mechanism is geometrical optics, and also that $\rho^{2}$ is large compared to the mean square first and second derivatives of $h(\theta, \varphi)$ with respect to $\theta$. This latter requirement implies that the surface is quasismooth in some sense.

We assume that the distant source is located in the direction of the unit polar vector $\mathbf{k}$. Then since the vectors $\mathbf{r}_{\theta}$ and $\mathbf{r}_{\varphi}$ are tangent to the surface the quantities

$$
\begin{gathered}
\xi=\mathbf{k} \cdot \mathbf{r}_{\theta}, \\
\eta=\mathbf{k} \cdot \mathbf{r}_{\varphi}
\end{gathered}
$$

should be practically zero at a specular point. We then have

$$
\begin{aligned}
& \xi=h_{\theta} \cos \theta-(\rho+h) \sin \theta, \\
& \eta=h_{\varphi} \cos \theta,
\end{aligned}
$$

and at a specular point

$$
\xi=\eta=0 .
$$

We wish to calculate the probability distribution of the event (9).

First we observe that

$$
\xi_{\varphi}=\eta_{\theta}
$$

Then following Longuet-Higgins, we consider the joint probability density of the quantities $\xi, \eta, \xi_{\theta}, \xi_{\varphi}, \eta_{\varphi}$, which we designate by

$$
P\left(\xi, \eta, \xi_{\theta}, \xi_{\varphi}, \eta_{\varphi}\right)
$$

This leads at once to the result

$$
P(\xi=\eta=0)=d \theta d \varphi \iiint P\left(0,0, \xi_{\theta}, \xi_{\varphi}, \eta_{\varphi}\right)\left|\xi_{\theta} \eta_{\varphi}-\xi_{\varphi}^{2}\right| d \xi_{\theta} d \xi_{\varphi} d \eta_{\varphi}
$$

for the probability of the occurrence of a specular point in an angular region of measure $d \theta d \varphi$.

Now since $P\left(\xi, \eta, \xi_{\theta}, \xi_{\varphi}, \eta_{\varphi}\right)$ is a multivariate gaussian distribution it will depend on the correlation matrix associated with the five variables, $\xi, \eta, \xi_{\theta}, \xi_{\varphi}, \eta_{\varphi}$. The elements of this matrix are cross correlations of the variables with $\theta=\theta^{\prime}$ and $\varphi=\varphi^{\prime}$. Some of the elements in the matrix vanish. we have

The correlations can be calculated (cf. [Swerling, 1962]) from (8) and (6). For example,

$$
\begin{aligned}
E(\xi \eta) & =\lim _{\substack{\theta^{\prime} \rightarrow \theta \\
\varphi^{\prime} \rightarrow \varphi}}\left\{E\left(h_{\theta}(\theta, \varphi) h_{\varphi^{\prime}}\left(\theta^{\prime}, \varphi^{\prime}\right)\right) \cos \theta \cos \theta^{\prime}-E\left(h(\theta, \varphi) h_{\varphi^{\prime}}\left(\theta^{\prime}, \varphi^{\prime}\right)\right) \sin \theta \cos \theta^{\prime}\right\} \\
= & \lim _{\substack{\theta^{\prime} \rightarrow \theta \\
\varphi^{\prime} \rightarrow \varphi}}\left\{\left[K^{\prime \prime}(\mu) \mu_{\theta} \mu_{\varphi^{\prime}}+K^{\prime}(\mu) \mu_{\varphi^{\prime}}\right] \cos \theta \cos \theta^{\prime}-K^{\prime}(\mu) \mu_{\varphi^{\prime}} \sin \theta \cos \theta^{\prime}\right\} \\
= & 0
\end{aligned}
$$


The matrix, reordered, can be written

$$
\left[\begin{array}{lllll}
E\left(\xi^{2}\right), & E\left(\xi \xi_{\theta}\right), & E\left(\xi \eta_{\varphi}\right), & 0, & 0 \\
E\left(\xi \xi_{\theta}\right), & E\left(\xi_{\theta}^{2}\right), & E\left(\xi_{\theta} \eta_{\varphi}\right), & 0, & 0 \\
E\left(\xi \eta_{\varphi}\right), & E\left(\xi_{\theta} \eta_{\varphi}\right), & E\left(\eta_{\varphi}^{2}\right), & 0, & 0 \\
0, & 0, & 0, & E\left(\eta^{2}\right), & E\left(\eta \xi_{\varphi}\right) \\
0, & 0, & 0, & E\left(\eta \xi_{\varphi}\right) & E\left(\xi_{\varphi}^{2}\right)
\end{array}\right)
$$

It follows that $P\left(\xi, \eta, \xi_{\theta}, \xi_{\varphi}, \eta_{\varphi}\right)$ is a product of two simpler distributions:

$$
P\left(\xi, \eta, \xi_{\theta}, \xi_{\varphi}, \eta_{\varphi}\right)=P\left(\xi, \xi_{\theta}, \eta_{\varphi}\right) P\left(\eta, \xi_{\varphi}\right) .
$$

Each of these is a multivariate gaussian distribution, itself. Then (10) takes the form

$$
P(\xi=\eta=0)=A d \theta d \varphi \int_{-\infty}^{\infty} \int_{-\infty}^{\infty} \int_{-\infty}^{\infty} \exp \left\{-\left(a \xi_{\theta}^{2}+2 b \xi_{\theta} \eta_{\varphi}+c \eta_{\varphi}^{2}+d \xi_{\varphi}^{2}\right)\right\}\left|\xi_{\theta} \eta_{\varphi}-\xi_{\varphi}^{2}\right| d \xi_{\theta} d \eta_{\varphi} d \xi_{\varphi}
$$

where the factor $A$ and the exponential coefficients $a, b, c, d$, are functions of $\theta$ and $\varphi$ which can be obtained from the correlation matrix.

To evaluate the integral in (12) we must break the region of integration up into regions in which $\left(\xi_{\theta} \eta_{\varphi}-\xi_{\varphi}^{2}\right)$ has a fixed sign. We have then,

$$
\begin{aligned}
& \int_{-\infty}^{\infty} \int_{-\infty}^{\infty} \int_{-\infty}^{\infty} \exp \{\left.-\left(a x^{2}+2 b x y+c y^{2}+d z^{2}\right)\right\}\left|x y-z^{2}\right| d z d x d y \\
&=4 \int_{0}^{\infty} \int_{-\infty}^{\infty} \int_{0}^{\infty}\left(z^{2}-x y\right) \exp \left\{-\left(a x^{2}+2 b x y+c y^{2}+d z^{2}\right)\right\} d z d x d y \\
& \quad+8 \int_{0}^{\infty} \int_{0}^{\infty} \int_{0}^{\sqrt{x y}}\left(x y-z^{2}\right) \exp \left\{-\left(a x^{2}+2 b x y+c y^{2}+d z^{2}\right)\right\} d z d x d y
\end{aligned}
$$

The first integral on the right in (13) offers no problem and can be evaluated explicitly. We have, in fact,

and

$$
\int_{0}^{\infty} \int_{-\infty}^{\infty} \int_{0}^{\infty} z^{2} \exp \left\{-\left(a x^{2}+2 b x y+c y^{2}+d z^{2}\right)\right\} d z d x d y=(\pi / d)^{\frac{2}{2}}\left(a c-b^{2}\right)^{-\frac{1}{2}} / 8,
$$

$$
\int_{0}^{\infty} \int_{-\infty}^{\infty} \int_{0}^{\infty} x y \exp \left\{-\left(a x^{2}+2 b x y+c y^{2}+d z^{2}\right)\right\} d z d x d y=-(b / 8)\left\{\pi /\left(a c-b^{2}\right)\right\}^{\frac{3}{2}} d^{-\frac{1}{2}}
$$

The second integral on the right of (13) can be obtained by differentiating the integral

$$
I(a, b, c, d)=\int_{0}^{\infty} \int_{0}^{\infty} \int_{0}^{\sqrt{x y}} \exp \left\{-\left(a x^{2}+2 b x y+c y^{2}+d z^{2}\right)\right\} d z d x d y
$$

with respect to $b$ and $d$. The integral on the right in (16) can be put into a formally simpler form. For this purpose, it is convenient to define

$$
F(s)=d^{-\frac{1}{2}} \operatorname{erf}\left(d^{\frac{1}{2}} s\right)=\int_{0}^{s} \exp \left(-d z^{2}\right) d z .
$$

We now change variables in (16), defining

$$
\begin{aligned}
& v=x^{2}, \\
& u=2 x y,
\end{aligned}
$$


and obtain

$$
\begin{aligned}
I & =\frac{1}{4} \int_{0}^{\infty} \int_{0}^{\infty} F(\sqrt{u / 2}) v^{-1} \exp \left\{-\left[a v+b u+c u^{2} /(4 v)\right]\right\} d v d u \\
& =\frac{1}{2} \int_{0}^{\infty} F(\sqrt{u / 2}) K_{0}(u \sqrt{a c}) \exp (-b u) d u .
\end{aligned}
$$

In (19) the function $K_{0}$ is a Macdonald function, which results from the identity

$$
\int_{0}^{\infty} \tau^{-1} \exp (-\alpha \tau-\beta / \tau) d \tau=2 K_{0}(2 \sqrt{\alpha \beta}), \quad \alpha>0, \beta>0 .
$$

We are interested in

$$
I_{1}=\frac{\partial I}{\partial b} \text { and } I_{2}=\frac{\partial I}{\partial d}
$$

If we collect the preceding results from (12) through (19) we obtain

$$
P(\xi=\eta=0)=A d \theta d \varphi\left\{\frac{1}{2}(\pi / d)^{\frac{3}{2}}\left(a c-b^{2}\right)^{-\frac{1}{2}}-b\left[\pi /\left(a c-b^{2}\right)\right]^{\frac{3}{2}} d^{-\frac{1}{2}} / 2+8 I_{2}-4 I_{1}\right\} .
$$

The parameters, $a, b, c, d$ are functions of $\theta$. They can be expressed in terms of the correlation matrix elements.

Let

$$
\Delta_{1}=\left|\begin{array}{lll}
E\left(\xi^{2}\right), & E\left(\xi \xi_{\theta}\right), & E\left(\xi \eta_{\varphi}\right) \\
E\left(\xi \xi_{\theta}\right), & E\left(\xi_{\theta}^{2}\right), & E\left(\xi_{\theta} \eta_{\varphi}\right) \\
E\left(\xi \eta_{\varphi}\right), & E\left(\xi_{\theta} \eta_{\varphi}\right), & E\left(\eta_{\varphi}^{2}\right)
\end{array}\right|
$$

and

$$
\Delta_{2}=\left|\begin{array}{ll}
E\left(\eta^{2}\right), & E\left(\eta \xi_{\varphi}\right) \\
E\left(\eta \xi_{\varphi}\right), & E\left(\xi_{\varphi}^{2}\right)
\end{array}\right| .
$$

Then

$$
\begin{aligned}
A & =(2 \pi)^{-\frac{5}{2}} \Delta_{1}^{-\frac{1}{2}} \Delta_{2}^{-\frac{1}{2}} \\
a & =\frac{1}{2}\left|\begin{array}{cc}
E\left(\xi^{2}\right), & E\left(\xi \eta_{\varphi}\right) \\
E\left(\xi \eta_{\varphi}\right), & E\left(\eta_{\varphi}^{2}\right)
\end{array}\right| \Delta_{1}^{-1}, \\
b & =-\frac{1}{2}\left|\begin{array}{cc}
E\left(\xi^{2}\right), & E\left(\xi \xi_{\theta}\right) \\
E\left(\xi \eta_{\varphi}\right), & E\left(\xi_{\theta} \eta_{\varphi}\right)
\end{array}\right| \Delta_{1}^{-1}, \\
c & =\frac{1}{2}\left|\begin{array}{cc}
E\left(\xi^{2}\right), & E\left(\xi \xi_{\theta}\right) \\
E\left(\xi \xi_{\theta}\right), & E\left(\xi_{\theta}^{2}\right)
\end{array}\right| \Delta_{1}^{-1}, \\
d & =\frac{1}{2} E\left(\eta^{2}\right) \Delta_{2}^{-1} .
\end{aligned}
$$

Let

$$
\begin{aligned}
& M_{0}=\sum_{n=0}^{\infty} W_{n} \\
& M_{1}=\sum_{n=0}^{\infty}\{n(n+1) / 2\} W_{n} \\
& M_{2}=\sum_{n=0}^{\infty}\{(n-1) n(n+1)(n+2) / 8\} W_{n} .
\end{aligned}
$$


Then we have

$$
\begin{aligned}
E\left(\xi^{2}\right) & =M_{1} \cos ^{2} \theta+\left(\rho^{2}+M_{0}\right) \sin ^{2} \theta, \\
E\left(\xi \xi_{\theta}\right) & =\frac{1}{2}\left(\rho^{2}+M_{0}-M_{1}\right) \sin 2 \theta, \\
E\left(\xi \eta_{\varphi}\right) & =-\left(M_{1} / 2\right) \sin 2 \theta \cos 2 \theta, \\
E\left(\eta^{2}\right) & =\left(M_{1} / 4\right) \sin ^{2} 2 \theta, \\
E\left(\eta \xi_{\varphi}\right) & =\left(M_{1} / 2\right) \sin 2 \theta \cos 2 \theta, \\
E\left(\xi_{\theta}^{2}\right) & =\left(3 M_{2}-M_{1}+M_{0}+\rho^{2}\right) \cos ^{2} \theta+4 M_{1}, \\
E\left(\xi_{\theta} \eta_{\varphi}\right) & =\left(M_{2} / 4+M_{1}\right) \sin ^{2} 2 \theta, \\
E\left(\xi_{\varphi}^{2}\right) & =M_{1}+\left(M_{2} / 4-M_{1}\right) \sin ^{2} 2 \theta, \\
E\left(\eta_{\varphi}^{2}\right) & =\frac{1}{4}\left(M_{2}+M_{1}\right) \sin ^{2} 2 \theta .
\end{aligned}
$$

We assume that $\rho^{2}$ is large compared to $M_{0}, M_{1}, M_{2}$ and that $\theta$ is not near $\pi / 2$. Then

where

$$
\begin{aligned}
\Delta_{1} & \sim M_{1} \rho^{2} \sin ^{2} 2 \theta \cos ^{2} \theta\left\{M_{2} \cos ^{2} \theta+M_{1} \sin ^{2} \theta-\left(M_{2}+2 M_{1}\right) \sin ^{2} \theta \cos 2 \theta\right\} / 4, \\
\Delta_{2} & =\left(M_{1} M_{2} \sin ^{4} 2 \theta\right) / 16, \\
a & \left.\sim\left\{M_{1} \cos ^{2} \theta+\rho \sin ^{2} \theta\right)\left(M_{1}+M_{2}\right)-M_{1}^{2} \cos ^{2} 2 \theta\right\} D^{-1}\left(2 M_{1} \rho^{2} \cos ^{2} \theta\right)^{-1}, \\
b & \sim-\left\{\left(M_{1} \cos ^{2} \theta+\rho^{2} \sin ^{2} \theta\right)\left(M_{2}+4 M_{1}\right)+\rho^{2} M_{1} \cos 2 \theta\right\} D^{-1}\left(2 M_{1} \rho^{2} \cos ^{2} \theta\right)^{-1}, \\
c & \sim 1 /\left(2 D \sin ^{2} \theta\right),
\end{aligned}
$$

$$
\begin{aligned}
& D=M_{2} \cos ^{2} \theta+M_{1} \sin ^{2} \theta-\left(M_{2}+2 M_{1}\right) \sin ^{2} \theta \cos 2 \theta, \\
& d=2 /\left(M_{2} \sin ^{2} 2 \theta\right) .
\end{aligned}
$$

From these results and (21) we find for the limiting case

that

$$
\rho \rightarrow \infty, \theta \rightarrow 0, \rho \sin \theta=0(1)
$$

Then

$$
a c=0(1), b=0(1), d \rightarrow \infty .
$$

and we have

$$
I_{2}=0\left(d^{-\frac{3}{2}}\right), I_{1}=0\left(d^{-\frac{1}{2}}\right),
$$

$$
P(\xi=\eta=0) \sim-A d \theta d \varphi\left\{b\left[\pi /\left(a c-b^{2}\right)\right]^{\frac{3}{2}} /\left(2 d^{\frac{1}{2}}\right)+4 I\right\},
$$

since the other terms are of higher order. Moreover, from (16) we have

where

$$
I_{1} \sim(\pi / d)^{\frac{1}{2}} \frac{\partial H}{\partial b} / 2
$$

$$
\begin{aligned}
H & =\int_{0}^{\infty} \int_{0}^{\infty} \exp \left\{-\left(a x^{2}+2 b x y+c y^{2}\right)\right\} d x d y \\
& =(a c)^{-\frac{1}{2}} \int_{0}^{\infty} \int_{0}^{\infty} \exp \left\{-\left[x^{2}+2 b(a c)^{-\frac{1}{2}} x y+y^{2}\right]\right\} d x d y .
\end{aligned}
$$

Let

$$
\xi=(a c)^{\frac{1}{2}}, \alpha=b(a c)^{-\frac{1}{2}} .
$$

Then

$$
\begin{aligned}
H & =\xi^{-1} \int_{0}^{\infty} \int_{0}^{\infty} \exp \left\{-\left(x^{2}+2 \alpha x y+y^{2}\right)\right\} d x d y \\
& =\xi^{-1}\left(1-\alpha^{2}\right)^{-\frac{1}{2}} \tan ^{-1}[(1-\alpha) /(1+\alpha)]^{\frac{1}{2}}
\end{aligned}
$$


where the integral can be evaluated, for example, by the use of polar coordinates.

We now have

$$
I_{1} \sim(1 / 2)(\pi / d)^{1 / 2}\left\{\left[b /\left(a c-b^{2}\right)^{3 / 2}\right] \tan ^{-1}[(\sqrt{a c}-b) /(\sqrt{a c}+b)]^{1 / 2}-(1 / 2)\left(a c-b^{2}\right)^{-1}\right\},
$$

and therefore

$$
\begin{aligned}
P(\xi=\eta=0) \sim-(16 \pi)^{-2} \Delta_{1}{ }^{-1 / 2} \Delta_{2}{ }^{-1 / 2}(2 / d)^{1 / 2}\left(a c-b^{2}\right)^{-3 / 2}\{\pi b & \\
& \left.+4 b \tan ^{-1}[(\sqrt{a c}-b) /(\sqrt{a c}+b)]^{1 / 2}-2\left(a c-b^{2}\right)^{1 / 2}\right\} d \theta d \varphi .
\end{aligned}
$$

We have for $\theta \sim 0$ :

$$
\begin{aligned}
& \Delta_{1} \sim M_{1} M_{2} \rho^{2} \sin ^{2} \theta, \\
& \Delta_{2} \sim M_{1} M_{2} \sin ^{4} \theta, \\
& a \sim\left\{\left(\rho^{2} \sin ^{2} \theta\right)\left(M_{2}+M_{1}\right)+M_{1} M_{2}\right\} /\left(2 M_{1} M_{2} \rho^{2}\right), \\
& b \sim-\left(2 M_{2}\right)^{-1} \\
& c \sim\left(2 M_{2} \sin ^{2} \theta\right)^{-1}, \\
& d \sim\left(2 M_{2} \sin ^{2} \theta\right)^{-1} \\
& a c \sim\left\{\rho^{2} \sin ^{2} \theta\left(M_{2}+M_{1}\right)+M_{1} M_{2}\right\} /\left(4 M_{1} M_{2}^{2} \rho^{2} \sin ^{2} \theta\right), \\
& a c-b^{2} \sim\left(\rho^{2} \sin ^{2} \theta+M_{1}\right) /\left(4 M_{1} M_{2} \rho^{2} \sin ^{2} \theta\right) .
\end{aligned}
$$

It may be observed from (31) and (30) that as $\theta$ approaches zero $P(\xi=\eta=0)$ becomes infinite. However, if we ask for the distribution $P_{\text {area }}$ of specular points per unit area instead, we shall have

$$
P_{\text {area }}=P(\xi=\eta=0) /\left(\rho^{2} \sin \theta d \theta d \varphi\right)
$$

which approaches a finite constant when $\theta$ approaches zero.

\section{Conclusions}

The distribution of specular points per unit area in the polar region, where $\theta$ is nearly zero (although the surface distance $\rho \theta$ may be of finite order), is given through expressions (30), (31), and (32) in terms of the surface spherical power spectral density moments $M_{1}$ and $M_{2}$. It may be observed by inspection that this distribution becomes indefinitely large if the moment $M_{2}$ grows indefinitely, and it approaches zero if the moment $M_{1}$ grows indefinitely. Since $M_{2}$ is related to surface curvature while $M_{1}$ is related to surface slope these observations are in accordance with geometrical intuition.

The case $\theta \sim \frac{\pi}{2}$ can be treated in a manner similar to the present one, but it is of less interest since the distribution will necessarily approach zero rapidly at those angles.

The authors thank R. Heimiller for his help in several discussions and some concrete suggestions which will have contributed materially to any success that this work may enjoy.

\section{References (Part 2)}

Longuet-Higgins, M. S. (1952), On the statistical distribution of the heights of sea-waves, J. Marine Res. 9, $245-266$.

Longuet-Higgins, M. S. (1956), Statistical properties of a moving wave-form, Proc. Cambridge Phil. Soc. 52, $234-245$. 
Longuet-Higgins M. S. (1957a), Statistical properties of an isotropic random surface, Phil. Trans. Roy. Soc. London A250, 157-174.

Longuet-Higgins, M. S. (1957b), The statistical analysis of a random moving surface, Phil. Trans. Roy. Soc. London A249, 321-387.

Longuet-Higgins, M. S. (1957c), On the velocities of the maxima in a moving wave-form, Proc. Cambridge Phil. Soc. 53, 230-233.

Rayleigh, Lord J. W. Strutt (1929), Theory of Sound, II, 89-96 (Macmillan and Co., Ltd., London).

Rice, S. O. (1951), Reflection of electromagnetic waves from slightly rough surfaces, Symposium on the Theory of Electromagnetic Waves, Interscience, New York, pp. 351.

Senior, T. B. A., and K. M. Siegel (1960), A theory of radar scattering by the moon, J. Res. NBS 64D (Radio Prop.) No. 3, 217-229.

Swerling, P. (1962), Statistical properties of the contours of random surfaces, IRE Trans. Inform. Theory IT-8, 315-321.

(Paper 68D9-405) 